\title{
Parameter Optimization of Single-Diode Model of Photovoltaic Cell Using Memetic Algorithm
}

\author{
Yourim Yoon ${ }^{1}$ and Zong Woo Geem ${ }^{2}$ \\ ${ }^{1}$ Department of Computer Engineering, Gachon University, 1342 Seongnam Daero, Seongnam 461-701, Republic of Korea \\ ${ }^{2}$ Department of Energy IT, Gachon University, 1342 Seongnam Daero, Seongnam 461-701, Republic of Korea \\ Correspondence should be addressed to Zong Woo Geem; zwgeem@gmail.com
}

Received 26 September 2015; Revised 5 November 2015; Accepted 11 November 2015

Academic Editor: Mahmoud M. El-Nahass

Copyright (c) 2015 Y. Yoon and Z. W. Geem. This is an open access article distributed under the Creative Commons Attribution License, which permits unrestricted use, distribution, and reproduction in any medium, provided the original work is properly cited.

This study proposes a memetic approach for optimally determining the parameter values of single-diode-equivalent solar cell model. The memetic algorithm, which combines metaheuristic and gradient-based techniques, has the merit of good performance in both global and local searches. First, 10 single algorithms were considered including genetic algorithm, simulated annealing, particle swarm optimization, harmony search, differential evolution, cuckoo search, least squares method, and pattern search; then their final solutions were used as initial vectors for generalized reduced gradient technique. From this memetic approach, we could further improve the accuracy of the estimated solar cell parameters when compared with single algorithm approaches.

\section{Introduction}

Determining the parameter values of photovoltaic (PV) cell models is very important when designing solar cells and estimating their performance. The key parameters that represent the behavior of solar cells include generated photocurrent, saturation current, series resistance, shunt resistance, and ideality factor [1]. Estimating these parameters accurately is essential for precise modeling and accurate performance evaluation of solar cells.

Several models have been proposed to describe the behavior of solar cells using current-voltage $(I-V)$ relationship [2-4]. The $I-V$ curve of a solar cell has nonlinear characteristics determined by the solar cell parameters. These models generally consist of analytical equations based on a physical description that formulate PV-generated current with the technical characteristics and the environmental variables including the operating voltage, the ambient temperature, and the irradiance [5]. Among numerous modeling approaches, the single-diode model (SDM) is the most widely utilized solar cell model in the literature. A general SDM includes five parameters: photocurrent, saturation current, diode ideality constant, series resistance, and shunt resistance.
So far various computational intelligence methods, such as genetic algorithm, particle swarm optimization, simulated annealing, and harmony search, have been proposed for optimal estimation of solar cell parameters. Many studies have aimed to overcome the shortcomings of the conventional deterministic algorithms and to investigate the efficiency and applicability of the algorithms. Hybrid methods combining two or more metaheuristic algorithms also have been applied to explore the capability of stochastic artificial intelligence algorithms in estimating solar cell parameters. These algorithms could find relevant parameter values by minimizing the root mean square error (RMSE) as the objective function in the optimization process.

Up to now, metaheuristic algorithms have shown a higher level of applicability in estimating solar cell parameters with fine performance. Nonetheless, we also presume that a memetic approach, which combines the well-developed evolutionary frameworks with gradient-based local search algorithm, can provide an opportunity for better solutions. Through this memetic combination, it can be seen that the accuracy represented by RMSE can be further improved because metaheuristic algorithm can be reinforced by calculus-based method in terms of local search performance and calculus-based method can be reinforced by 
metaheuristic algorithm in terms of global search performance.

This paper is organized as follows. Optimization formulation of the problem to determine the solar cell parameters is described in Section 2. In Section 3, a memetic implementation, which integrates gradient-based local search into the evolutionary frameworks for better determining the parameters, is described. Then, simulation results of the proposed approach are provided in Section 4, followed by conclusions in Section 5.

\section{Optimization Formulation of Photovoltaic Model}

Solar cells are made of various semiconductor materials. The working mechanism of solar cells is essentially based on the PV effect, which is the generation of a potential difference at the $\mathrm{P}-\mathrm{N}$ junction in regard to visible or other radiations. When a solar cell is exposed to light source, the semiconductor materials capture photons, and then charged carriers are generated. Potential difference and current in the external circuit guide to the separation of carriers in the internal electric field created by the junction and collection at the electrodes. The photogenerated charge carriers can be accordingly captured in the form of an electric current, that is, $I_{\mathrm{ph}}$. Eliminating this effect, a solar cell behaves like a traditional diode that does not rely on any light parameters. The Shockley diode equation is utilized to represent the current flowing through the diode $\left(I_{D}\right)$ [5] as follows:

$$
I_{D}=I_{\mathrm{SD}}\left(e^{V_{d} / n V_{t}}-1\right)
$$

where $I_{\mathrm{SD}}$ denotes diode saturation current (normal diode current), $V_{d}$ denotes electrical potential difference between both ends of the diode, $n$ denotes ideality factor, and $V_{t}$ denotes thermal voltage. Here, the thermal voltage can be represented as follows:

$$
V_{t}=\frac{k T}{q}
$$

where $k$ denotes Boltzmann constant $\left(1.380650 \times 10^{-23} \mathrm{~J} / \mathrm{K}\right)$, $T$ denotes ambient temperature ( $306 \mathrm{~K}$ in this study), and $q$ denotes electron charge $\left(1.602176 \times 10^{-19} \mathrm{C}\right)$.

SDM, which can be visually described in Figure 1, follows the superposition principle [6-8]. Thus, the terminal current $I$ is equal to $I_{\mathrm{ph}}$ subtracting the currents diverting through the diode and resistance as follows:

$$
\begin{aligned}
I & =I_{\mathrm{ph}}-I_{D}-I_{\mathrm{sh}} \\
& =I_{\mathrm{ph}}-I_{\mathrm{SD}}\left(e^{\left(V+I R_{s}\right) / n V_{t}}-1\right)-\frac{V+I R_{s}}{R_{\mathrm{sh}}},
\end{aligned}
$$

where $I_{\text {sh }}$ denotes shunt current, $V$ denotes operating voltage, $R_{s}$ denotes series resistance, and $R_{\mathrm{sh}}$ denotes shunt resistance.

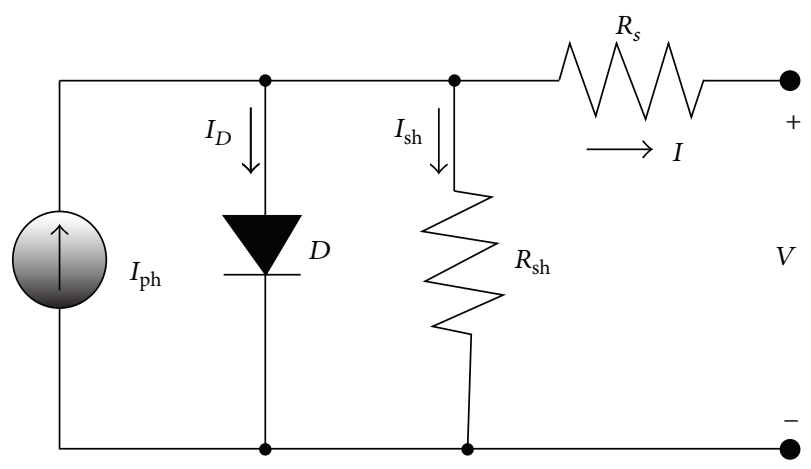

FIGURE 1: Single-diode-equivalent circuit model of solar cell.

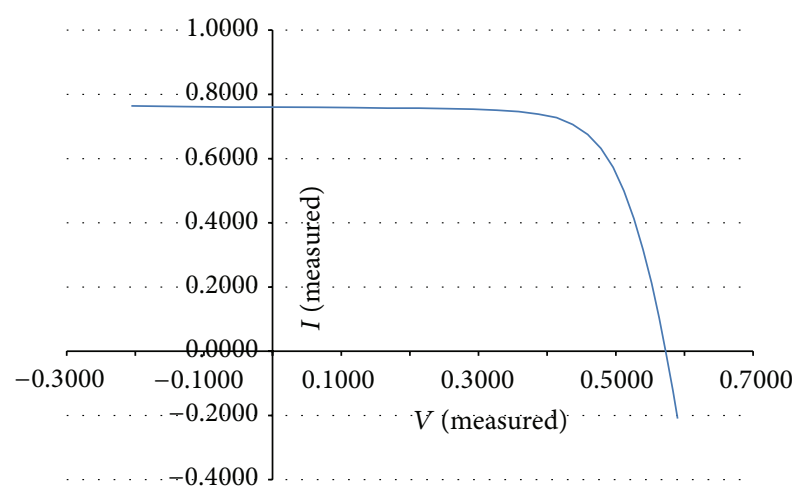

Figure 2: Experimental $I-V$ curve of a PV module.

Figure 1 can be transformed into an optimization problem with respect to RMSE once measured data of $I-V$ relationship is given, as follows:

$$
\text { Minimize } \sqrt{\frac{1}{N} \sum_{i=1}^{N}\left(I_{\text {measured }}-I_{\text {calculated }}\left(I_{\mathrm{ph}}, I_{\mathrm{SD}}, n, R_{s}, R_{\mathrm{sh}}\right)\right)^{2}} .
$$

This optimization problem has five decision variables of cell-generated photocurrent $I_{\mathrm{ph}}$, diode saturation current $I_{\mathrm{SD}}$, diode ideality factor $n$, series resistance $R_{s}$, and shunt resistance $R_{\mathrm{sh}}$. The measured $I-V$ data is given in Table 1 [5] where the first column represents measured voltage data and the second column represents measured current data, which originally came from a commercial (RTC France) $57 \mathrm{~mm}$ diameter silicon solar cell performing at the standard irradiance level $\left(1\right.$ sun $\left(1000 \mathrm{~W} / \mathrm{m}^{2}\right)$ at $\left.306^{\circ} \mathrm{K}\right)$ [9]. Also, the $I-V$ data is graphically shown in Figure 2.

\section{Memetic Implementation}

Memetic computing is a branch in computer science which considers complicate structures as the combination of heterogeneous operators, named memes, whose evolutionary interactions contribute to intelligent structures for problem solving $[10,11]$. For recent decades, memetic algorithms have been widely applied in the large-scale complex optimization problems. 
TABLE 1: Measured $I-V$ data and computation results.

\begin{tabular}{|c|c|c|c|c|c|c|c|c|c|c|c|}
\hline \multirow{2}{*}{$V$} & \multirow[t]{2}{*}{$I$} & \multicolumn{10}{|c|}{ Algorithm } \\
\hline & & SA & GA2 & PS & NLS1 & PSO & LS & NLS2 & HS & RADE & $\mathrm{CS}$ \\
\hline-0.2057 & 0.7640 & 0.7662 & 0.7662 & 0.7645 & 0.7641 & 0.7641 & 0.7637 & 0.7645 & 0.7640 & 0.7641 & 0.7641 \\
\hline-0.1291 & 0.7620 & 0.7644 & 0.7644 & 0.7633 & 0.7627 & 0.7627 & 0.7624 & 0.7629 & 0.7626 & 0.7627 & 0.7627 \\
\hline-0.0588 & 0.7605 & 0.7628 & 0.7628 & 0.7622 & 0.7614 & 0.7614 & 0.7612 & 0.7615 & 0.7613 & 0.7614 & 0.7614 \\
\hline 0.0057 & 0.7605 & 0.7613 & 0.7612 & 0.7612 & 0.7602 & 0.7602 & 0.7601 & 0.7602 & 0.7601 & 0.7602 & 0.7602 \\
\hline 0.0646 & 0.7600 & 0.7599 & 0.7598 & 0.7603 & 0.7591 & 0.7591 & 0.7592 & 0.7590 & 0.7590 & 0.7591 & 0.7591 \\
\hline 0.1185 & 0.7590 & 0.7586 & 0.7585 & 0.7595 & 0.7581 & 0.7581 & 0.7583 & 0.7579 & 0.7580 & 0.7580 & 0.7581 \\
\hline 0.1678 & 0.7570 & 0.7574 & 0.7573 & 0.7586 & 0.7571 & 0.7571 & 0.7574 & 0.7569 & 0.7570 & 0.7571 & 0.7571 \\
\hline 0.2132 & 0.7570 & 0.7563 & 0.7561 & 0.7577 & 0.7562 & 0.7562 & 0.7565 & 0.7559 & 0.7561 & 0.7561 & 0.7562 \\
\hline 0.2545 & 0.7555 & 0.7550 & 0.7547 & 0.7566 & 0.7551 & 0.7551 & 0.7556 & 0.7547 & 0.7550 & 0.7551 & 0.7551 \\
\hline 0.2924 & 0.7540 & 0.7532 & 0.7529 & 0.7550 & 0.7537 & 0.7537 & 0.7542 & 0.7532 & 0.7536 & 0.7537 & 0.7537 \\
\hline 0.3269 & 0.7505 & 0.7506 & 0.7500 & 0.7522 & 0.7515 & 0.7515 & 0.7520 & 0.7507 & 0.7513 & 0.7514 & 0.7514 \\
\hline 0.3585 & 0.7465 & 0.7460 & 0.7452 & 0.7472 & 0.7475 & 0.7475 & 0.7480 & 0.7463 & 0.7473 & 0.7473 & 0.7473 \\
\hline 0.3873 & 0.7385 & 0.7379 & 0.7368 & 0.7386 & 0.7403 & 0.7403 & 0.7407 & 0.7387 & 0.7401 & 0.7401 & 0.7401 \\
\hline 0.4137 & 0.7280 & 0.7240 & 0.7227 & 0.7238 & 0.7278 & 0.7278 & 0.7278 & 0.7253 & 0.7274 & 0.7272 & 0.7273 \\
\hline 0.4373 & 0.7065 & 0.7020 & 0.7008 & 0.7011 & 0.7077 & 0.7077 & 0.7072 & 0.7040 & 0.7069 & 0.7067 & 0.7067 \\
\hline 0.4590 & 0.6755 & 0.6683 & 0.6677 & 0.6668 & 0.6766 & 0.6766 & 0.6751 & 0.6713 & 0.6751 & 0.6748 & 0.6748 \\
\hline 0.4784 & 0.6320 & 0.6213 & 0.6220 & 0.6199 & 0.6329 & 0.6328 & 0.6299 & 0.6256 & 0.6303 & 0.6300 & 0.6300 \\
\hline 0.4960 & 0.5730 & 0.5595 & 0.5622 & 0.5590 & 0.5751 & 0.5751 & 0.5703 & 0.5657 & 0.5710 & 0.5708 & 0.5708 \\
\hline 0.5119 & 0.4990 & 0.4838 & 0.4886 & 0.4848 & 0.5042 & 0.5042 & 0.4969 & 0.4924 & 0.4981 & 0.4979 & 0.4979 \\
\hline 0.5265 & 0.4130 & 0.3937 & 0.4007 & 0.3970 & 0.4199 & 0.4199 & 0.4098 & 0.4057 & 0.4114 & 0.4114 & 0.4114 \\
\hline 0.5398 & 0.3165 & 0.2928 & 0.3011 & 0.2985 & 0.3257 & 0.3257 & 0.3122 & 0.3092 & 0.3144 & 0.3145 & 0.3145 \\
\hline 0.5521 & 0.2120 & 0.1818 & 0.1902 & 0.1899 & 0.2225 & 0.2225 & 0.2054 & 0.2038 & 0.2082 & 0.2084 & 0.2084 \\
\hline 0.5633 & 0.1035 & 0.0654 & 0.0721 & 0.0754 & 0.1149 & 0.1148 & 0.0939 & 0.0941 & 0.0974 & 0.0976 & 0.0978 \\
\hline 0.5736 & -0.0100 & -0.0529 & -0.0504 & -0.0422 & 0.0063 & 0.0062 & -0.0186 & -0.0163 & -0.0144 & -0.0142 & -0.0140 \\
\hline 0.5833 & -0.1230 & -0.1782 & -0.1819 & -0.1673 & -0.1080 & -0.1081 & -0.1371 & -0.1323 & -0.1319 & -0.1319 & -0.1316 \\
\hline 0.5900 & -0.2100 & -0.2680 & -0.2785 & -0.2583 & -0.1891 & -0.1893 & -0.2212 & -0.2145 & -0.2153 & -0.2155 & -0.2151 \\
\hline & & 0.02165 & 0.02158 & 0.01741 & 0.00719 & 0.00715 & 0.00477 & 0.00474 & 0.00280 & 0.00278 & 0.00269 \\
\hline
\end{tabular}

The problem of balance between global and local search, that is, balance between computational intelligence and gradient-based search algorithm, has been explored under a multiobjective optimization setting in $[12,13]$. In [14], a memetic algorithm was presented to analyze the evolutionary artificial neural network for training of a medical application. For a large-scale combinatorial optimization problem, a parallel memetic algorithm with selective local search was proposed in [15]. In [16-18], memetic algorithms were applied to solve scheduling and planning problems such as vehicle routing and path planning. A fast adaptive memetic algorithm for a design of controller of engineering drives was presented in [19], and a compact memetic differential algorithm has been developed for a robot control in [20]. In [21], a differential evolution-based hybrid algorithm was provided to solve the optimization problem in flow-dynamic design. In [22] a novel memetic algorithm scheme was proposed by combining metaheuristic algorithm and gradientbased technique to find better solutions for an economic load dispatch problem with valve-point loading.

In order to explore the opportunity of better solution in determining solar cell parameters using the memetic combination proposed in this study, various metaheuristic algorithms, such as genetic algorithm, simulated annealing, particle swarm optimization, harmony search, and cuckoo search, as well as mathematical approaches, such as least squares method and pattern search, have been performed, which have shown the good level of performances among all the existing relevant approaches in literature.

Once good parameter values for the PV cell model are obtained using the above-mentioned various algorithms, the values can be further polished using gradient-based techniques. In this study, we adopted generalized reduced gradient (GRG) method as the gradient-based local search technique.

The GRG algorithm was first developed by Abadie and Carpentier [23] as an extension of the reduced gradient method to solve a general constrained nonlinear programming problem, which can be stated as follows [24]:

$$
\begin{array}{cl}
\text { Minimize } & f(\mathbf{x}), \quad \mathbf{x} \in F \subseteq S \subseteq R^{n} \\
\text { subject to } & h_{i}(\mathbf{x})=0, \quad i=1, \ldots, p \\
& g_{j}(\mathbf{x}) \leq 0, \quad j=p+1, \ldots, q \\
& a_{k} \leq x_{k} \leq b_{k}, \quad k=1, \ldots, n,
\end{array}
$$


where $\mathbf{x}=\left(x_{1}, x_{2}, \ldots, x_{n}\right)$ is a vector of $n$ variables, $f(\mathbf{x})$ is the objective function, $h_{i}(\mathbf{x})$ is the $i$ th equality constraint, and $g_{j}(\mathbf{x})$ is the $j$ th inequality constraint $(q<n) . S$ is the whole search space and $F$ is the feasible search space. The $a_{k}$ and $b_{k}$ denote the lower and upper bounds of the variable $x_{k}$, respectively. It is assumed that all problem functions $f(\mathbf{x})$, $h_{i}(\mathbf{x})$, and $g_{j}(\mathbf{x})$ are twice continuously differentiable.

GRG transforms inequality constraints into equality constraints by introducing slack variables. Hence all the constraints are of equality form and can be represented as follows:

$$
h_{i}(\mathbf{x})=0, \quad i=1, \ldots, p,
$$

where $\mathbf{x}$ contains both original variables and slacks. Variables are divided into dependent variables $\mathbf{x}_{D}$ and independent ones $\mathbf{x}_{I}$ (or basic and nonbasic variables, resp.):

$$
\mathbf{x}=\left[\begin{array}{c}
\mathbf{x}_{D} \\
\vdots \\
\mathbf{x}_{I}
\end{array}\right]
$$

The names of basic and nonbasic variables are from linear programming. Similarly, the gradient of the objective function, bounds, and the Jacobian matrix may be partitioned as follows:

$$
\begin{aligned}
\mathbf{a} & =\left[\begin{array}{c}
\mathbf{a}_{D} \\
\vdots \\
\mathbf{a}_{I}
\end{array}\right], \\
\mathbf{b} & =\left[\begin{array}{c}
\mathbf{b}_{D} \\
\vdots \\
\mathbf{b}_{I}
\end{array}\right], \\
\nabla f(\mathbf{x}) & =\left[\begin{array}{c}
\nabla_{D} f(\mathbf{x}) \\
\vdots \\
\nabla_{I} f(\mathbf{x})
\end{array}\right], \\
J(\mathbf{x}) & =\left[\begin{array}{ccc}
\nabla_{D} h_{1}(\mathbf{x}) & \cdots & \nabla_{I} h_{1}(\mathbf{x}) \\
& \vdots \\
\nabla_{D} h_{p}(\mathbf{x}) & \cdots & \nabla_{I} h_{p}(\mathbf{x})
\end{array}\right] .
\end{aligned}
$$

Let $\mathbf{x}^{0}$ be an initial feasible solution, which satisfies equality constraints and bound constraints. Note that basic variables must be selected so that $J_{D}\left(\mathbf{x}^{0}\right)$ is nonsingular.

The reduced gradient vector is determined as follows:

$$
\mathbf{g}_{I}=\nabla_{I} f\left(\mathbf{x}^{0}\right)-\nabla_{D} f\left(\mathbf{x}^{0}\right)\left(J_{D}\left(\mathbf{x}^{0}\right)\right)^{-1} J_{I}\left(\mathbf{x}^{0}\right) .
$$

The search directions for the independent and the dependent variables are given by

$$
\begin{aligned}
& \mathbf{d}_{I}= \begin{cases}0, & \text { if } x_{i}^{0}=a_{i}, g_{i}>0 \\
0, & \text { if } x_{i}^{0}=b_{i}, g_{i}<0 \\
-g_{i}, & \text { otherwise, }\end{cases} \\
& \mathbf{d}_{D}=-\left(J_{D}\left(\mathbf{x}^{0}\right)\right)^{-1} J_{I}\left(\mathbf{x}^{0}\right) \mathbf{d}_{I} .
\end{aligned}
$$

A line search is performed to find the step length $\alpha$ as the solution to the following problem:

$$
\begin{array}{ll}
\text { Minimize } & f\left(\mathbf{x}^{0}+\alpha \mathbf{d}\right) \\
\text { subject to } & 0 \leq \alpha \leq \alpha_{\max } \\
& \alpha_{\max }=\operatorname{sub}\left\{\frac{\alpha}{\mathbf{a}} \leq \mathbf{x}^{0} \leq\left(\mathbf{x}^{0}+\alpha \mathbf{d}\right) \leq \mathbf{b}\right\} .
\end{array}
$$

The optimal solution $\alpha^{*}$ to the problem gives the next solution:

$$
\mathbf{x}^{1}=\mathbf{x}^{0}+\alpha^{*} \mathbf{d}
$$

A more detailed description of the GRG method can be found in [25].

\section{Computational Results}

For this PV parameter optimization problem, various metaheuristic and mathematical algorithms have so far proposed their solutions, as summarized in Table 2. As seen in Table 2, the obtained solution vector of PV model parameters from simulated annealing (SA) [26] made RMSE of 0.02165, which is the worst one among various solution vectors if outlier from genetic algorithm 1 (GA1) is not considered; genetic algorithm 2 (GA2) [27] produced RMSE of 0.02158, which is slightly better than SA; pattern search (PS) [28] produced RMSE of 0.01741; nonlinear least squares 1 (NLS1) [9] produced RMSE of 0.00719; particle swarm optimization (PSO) [4] produced RMSE of 0.00715; least squares (LS) method [29] produced RMSE of 0.00477; nonlinear least squares 2 (NLS2) [1] produced RMSE of 0.00474; harmony search (HS) [30] produced RMSE of 0.00280; repaired adaptive differential evolution (RADE) [31] produced RMSE of 0.00278; and cuckoo search (CS) [5] produced RMSE of 0.00269 which is the best one among algorithms. Ye et al. [4] proposed GA1, but it does not appear to be good because its RMSE is huge (132,590). Although there are two nonlinear least squares (NLS1 and NLS2) and two genetic algorithms (GA1 and GA2), they are different in terms of algorithm structure and algorithm parameter values.

The search range of decision variable is $I_{\mathrm{ph}}=[0,1], I_{\mathrm{SD}}=$ $[0,1 e-6], R_{s}=[0,0.5], R_{\mathrm{sh}}=[0,100]$, and $n=[1,2][30]$, and several metaheuristic algorithms such as HS, RADE, and CS provided a number of function evaluations ( $\mathrm{HS}=5,000$; RADE $=10,000$; and CS $=5,000)$ while PSO provided time taken (242 seconds) and SA and GA2 did not mention any. 
TABLE 2: Optimal parameter values from various algorithms.

\begin{tabular}{|c|c|c|c|c|c|c|c|c|c|c|c|}
\hline \multirow{2}{*}{ Parameter } & \multicolumn{11}{|c|}{ Algorithm } \\
\hline & SA & GA2 & PS & NLS1 & PSO & LS & NLS2 & HS & RADE & CS & GA1 \\
\hline$I_{\mathrm{ph}}$ & 0.7620 & 0.7619 & 0.7617 & 0.7608 & 0.7608 & 0.7607 & 0.7609 & 0.7607 & 0.7608 & 0.7608 & 0.7756 \\
\hline$I_{\mathrm{SD}}$ & $4.8 \times 10^{-7}$ & $8.1 \times 10^{-7}$ & $10.0 \times 10^{-7}$ & $3.2 \times 10^{-7}$ & $3.2 \times 10^{-7}$ & $3.3 \times 10^{-7}$ & $4.0 \times 10^{-7}$ & $3.0 \times 10^{-7}$ & $3.2 \times 10^{-7}$ & $3.2 \times 10^{-7}$ & $5.6 \times 10^{-7}$ \\
\hline$R_{s}$ & 0.0345 & 0.0299 & 0.0313 & 0.0364 & 0.0364 & 0.0364 & 0.0364 & 0.0366 & 0.0364 & 0.0364 & 0.0697 \\
\hline$R_{\mathrm{sh}}$ & 43.103 & 42.373 & 64.103 & 53.763 & 53.796 & 60.241 & 49.505 & 53.595 & 53.7185 & 53.7185 & 10.084 \\
\hline$n$ & 1.5172 & 1.5751 & 1.6000 & 1.4837 & 1.4838 & 1.4816 & 1.5039 & 1.4754 & 1.4812 & 1.4812 & 0.8053 \\
\hline RMSE & 0.02165 & 0.02158 & 0.01741 & 0.00719 & 0.00715 & 0.00477 & 0.00474 & 0.00280 & 0.00278 & 0.00269 & 132590 \\
\hline
\end{tabular}

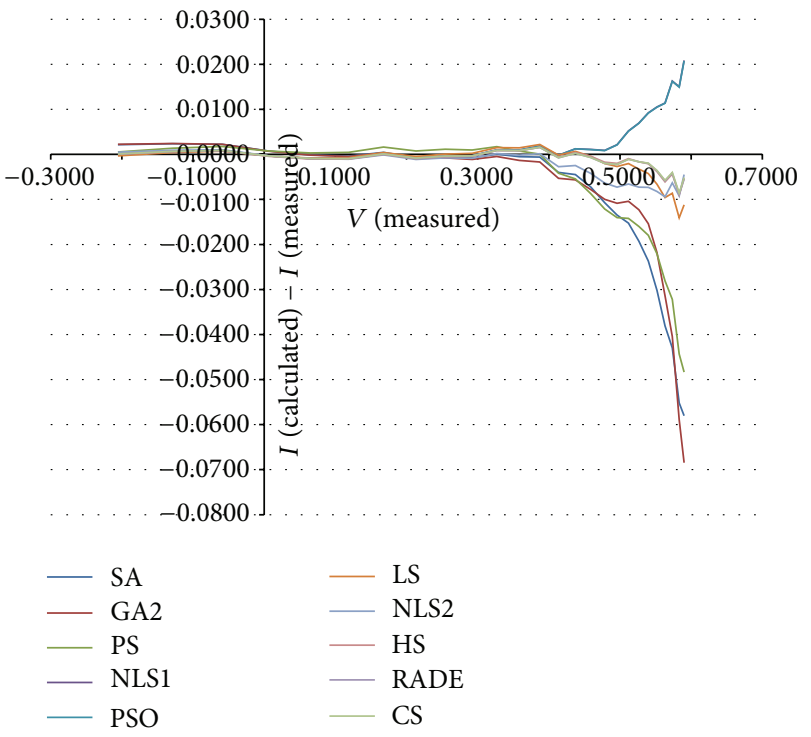

FIGURE 3: Errors between calculated and measured currents (single algorithm).

Table 1 shows corresponding currents calculated by various algorithms, and Figure 3 shows the errors between calculated and measured currents from various algorithms. As observed in the figure, the magnitude of the errors abruptly increases after around $0.4 \mathrm{~V}$. Presumably this is because representing rapid change is more difficult than representing monotonic change. The measured current abruptly drops after around $0.4 \mathrm{~V}$ as seen in Figure 2.

The above-enumerated algorithms found good PV parameter values with small RMSE. However, is it possible to further improve the solution quality? The memetic algorithm, which combines metaheuristic and gradientbased algorithms, may answer to this question because the memetic algorithm can complement the weakness of metaheuristic algorithm by using gradient-based algorithm. Thus, we can obtain even better solutions if we introduce gradient-based algorithm to the metaheuristic algorithm.

The gradient-based algorithm (GRG in this study) by itself cannot find good solution unless it starts with good and feasible initial vector. Otherwise, it will get stuck in a local optimum or will diverge instead of converging.

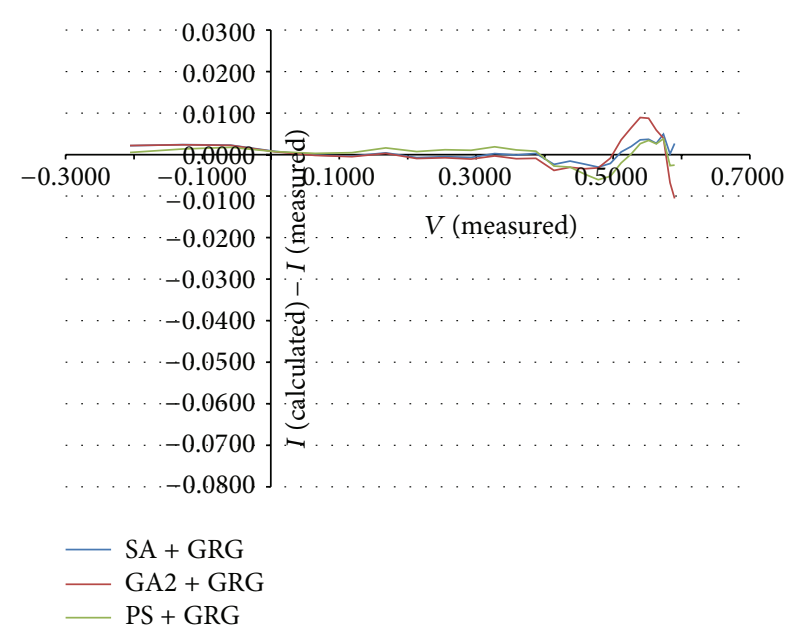

FIGURE 4: Errors between calculated and measured currents (memetic approach).

For this PV parameter optimization problem also, finding good solutions using gradient-based algorithm requires proper feasible initial vector, which is not very easy and tedious task. Thus, the result vectors from metaheuristic or other mathematical algorithms can be used as the proper initial vectors for the gradient-based algorithm.

The results from the memetic approach were tabulated in Table 3. As seen in the table, some initial vectors could be further improved while others could not because of getting stuck in local optimal (LO). SA + GRG could improve RMSE from 0.02165 to 0.00209 ; GA2 + GRG could improve RMSE from 0.02158 to 0.00432 ; PS + GRG could improve RMSE from 0.01741 to 0.00261 ; however, NLS1 + GRG, PSO + GRG, $\mathrm{LS}+\mathrm{GRG}, \mathrm{NLS} 2$ + GRG, HS + GRG, RADE + GRG, and CS + GRG could not improve RMSE any further because they already reached local optima where there is no room to escape using the local search technique. Here, the computation time of GRG part is less than one second, so it does not become a burden.

Figure 4 shows the errors between calculated and measured currents from various memetic approaches. As observed in the figure, the magnitude of the errors is less than single algorithm approach. While CS found the best RMSE of 0.00269 in single algorithm approach, PS + GRG 
TABLE 3: Optimal parameter values from memetic algorithms.

\begin{tabular}{|c|c|c|c|c|c|c|c|c|c|c|}
\hline \multirow[b]{2}{*}{ Parameter } & \multicolumn{10}{|c|}{ Algorithm } \\
\hline & $\begin{aligned} & \text { SA } \\
+ & \text { GRG }\end{aligned}$ & $\begin{array}{c}\text { GA2 } \\
+ \text { GRG }\end{array}$ & $\begin{aligned} & \text { PS } \\
+ & \text { GRG }\end{aligned}$ & NLS1 + GRG & $\mathrm{PSO}+\mathrm{GRG}$ & $\mathrm{LS}+\mathrm{GRG}$ & NLS2 + GRG & $\mathrm{HS}+\mathrm{GRG}$ & RADE + GRG & $\mathrm{CS}+\mathrm{GRG}$ \\
\hline$I_{\mathrm{ph}}$ & 0.7620 & 0.7619 & 0.7617 & & & & & & & \\
\hline$I_{\mathrm{SD}}$ & $4.5 \times 10^{-7}$ & $7.6 \times 10^{-7}$ & $9.5 \times 10^{-7}$ & & & & & & & \\
\hline$R_{s}$ & 0.0345 & 0.0301 & 0.0313 & LO & LO & LO & $\mathrm{LO}$ & LO & LO & $\mathrm{LO}$ \\
\hline$R_{\mathrm{sh}}$ & 43.103 & 42.373 & 64.103 & & & & & & & \\
\hline$n$ & 1.5172 & 1.5749 & 1.6000 & & & & & & & \\
\hline RMSE & 0.00209 & 0.00432 & 0.00261 & & & & & & & \\
\hline
\end{tabular}

TABLE 4: Sensitivity analysis of ambient temperature.

\begin{tabular}{lcccccc}
\hline Celsius $\left({ }^{\circ} \mathrm{C}\right)$ & Kelvin $\left({ }^{\circ} \mathrm{K}\right)$ & RMSE & $I_{\mathrm{ph}}$ & $I_{\mathrm{SD}}$ & $R_{s}$ & $R_{\mathrm{sh}}$ \\
\hline-5 & 268.15 & 0.01438 & 0.7776 & $1.72 E-07$ & 0.0397 & 43.1035 \\
0 & 273.15 & 0.01658 & 0.7509 & $2.37 E-07$ & 0.0293 & 43.1034 \\
5 & 278.15 & 0.00520 & 0.7666 & $2.05 E-07$ & 0.0400 & 43.1033 \\
10 & 283.15 & 0.01107 & 0.7756 & $2.78 E-07$ & 0.0400 & 43.1033 \\
15 & 288.15 & 0.01454 & 0.7802 & $2.93 E-07$ & 0.0403 & 43.1032 \\
20 & 293.15 & 0.01952 & 0.7620 & $2.30 E-07$ & 0.0345 & 43.1034 \\
25 & 298.15 & 0.00391 & 0.7620 & $3.10 E-07$ & 0.0345 & 43.1034 \\
30 & 303.15 & 0.00203 & 0.7620 & $3.96 E-07$ & 0.0345 & 1.5808 \\
35 & 308.15 & 0.01491 & 0.7620 & $4.80 E-07$ & 0.0345 & 1.5172 \\
40 & 313.15 & 0.00236 & 0.7626 & $6.27 E-07$ & 0.0331 & 43.1034 \\
\hline
\end{tabular}

could find the better RMSE of 0.00261 , and SA + GRG could find even better RMSE of 0.00209 .

While the above approach used the constant ambient temperature $\left(306^{\circ} \mathrm{K}\right.$ or $\left.32.85^{\circ} \mathrm{C}\right)$, the sensitivity of the ambient temperature was also tested under various operation (weather) conditions between $-5^{\circ} \mathrm{C}$ and $40^{\circ} \mathrm{C}$. SA + GRG produced the computation results as shown in Table 4. As seen in the table, $I_{\mathrm{ph}}$ and $R_{s}$ are fluctuating, $I_{\mathrm{SD}}$ is increasing, $n$ is decreasing, and $R_{\text {sh }}$ is almost constant.

\section{Conclusions}

In this study, a memetic approach has proposed for optimally determining the parameter values of single-diode-equivalent solar cell model. The memetic approach considered various metaheuristic and mathematical algorithms and combined them with GRG technique. The results validated the performance of the memetic approach, which further improved the quality of solutions originally obtained by various algorithms.

The proposed memetic approach in this work can be also further applied to more complicated and realistic PV models with various module types [32] in the future. And theoretically the balance between global search and local search can be a good future research topic because too much exploited solutions do not appear to be good initial vectors for local search algorithms. In addition, parameter-setting-free technique $[33,34]$ can be also incorporated in metaheuristic approach in order to eliminate tedious algorithm parameter setting task.

\section{Nomenclature}

I: Observed terminal current

$I_{D}$ : Diode current

$I_{\mathrm{ph}}$ : Cell-generated photocurrent

$I_{\mathrm{SD}}$ : Diode saturation current

$I_{\text {sh }}$ : Shunt current

$k$ : Boltzmann constant $\left(1.380650 \times 10^{-23} \mathrm{~J} / \mathrm{K}\right)$

$n$ : Diode ideality factor

$q$ : $\quad$ Electron charge $\left(1.602176 \times 10^{-19} \mathrm{C}\right)$

$R_{s}$ : Series resistance

$R_{\text {sh }}$ : Shunt resistance

T: Ambient temperature (306 K in this study)

$V$ : Observed operation voltage

$V_{d}$ : Electrical potential difference between both ends of the diode

$V_{t}$ : Thermal voltage.

\section{Conflict of Interests}

The authors declare that there is no conflict of interests regarding the publication of this paper.

\section{Acknowledgment}

This research was supported by the Gachon University research fund of 2015 (GCU-2015-0030). 


\section{References}

[1] M. Chegaar, Z. Ouennoughi, and A. Hoffmann, "A new method for evaluating illuminated solar cell parameters," Solid-State Electronics, vol. 45, no. 2, pp. 293-296, 2001.

[2] M. Chegaar, Z. Ouennoughi, F. Guechi, and H. Langueur, "Determination of solar cells parameters under illuminated conditions," Journal of Electron Devices, vol. 2, pp. 17-21, 2003.

[3] W. Xiao, M. G. J. Lind, W. G. Dunford, and A. Capel, "Real-time identification of optimal operating points in photovoltaic power systems," IEEE Transactions on Industrial Electronics, vol. 53, no. 4, pp. 1017-1026, 2006.

[4] M. Ye, X. Wang, and Y. Xu, "Parameter extraction of solar cells using particle swarm optimization," Journal of Applied Physics, vol. 105, no. 9, Article ID 094502, 2009.

[5] J. Ma, T. O. Ting, K. L. Man, N. Zhang, S.-U. Guan, and P. W. H. Wong, "Parameter estimation of photovoltaic models via cuckoo search," Journal of Applied Mathematics, vol. 2013, Article ID 362619, 8 pages, 2013.

[6] V. Lo Brano, A. Orioli, G. Ciulla, and A. Di Gangi, "An improved five-parameter model for photovoltaic modules," Solar Energy Materials and Solar Cells, vol. 94, no. 8, pp. 1358-1370, 2010.

[7] W. De Soto, S. A. Klein, and W. A. Beckman, "Improvement and validation of a model for photovoltaic array performance," Solar Energy, vol. 80, no. 1, pp. 78-88, 2006.

[8] M. G. Villalva, J. R. Gazoli, and E. R. Filho, "Comprehensive approach to modeling and simulation of photovoltaic arrays," IEEE Transactions on Power Electronics, vol. 24, no. 5, pp. 11981208, 2009.

[9] T. Easwarakhanthan, J. Bottin, I. Bouhouch, and C. Boutrit, "Nonlinear minimization algorithm for determining the solar cell parameters with microcomputers," International Journal of Solar Energy, vol. 4, no. 1, pp. 1-12, 1986.

[10] G. Iacca, F. Neri, E. Mininno, Y.-S. Ong, and M.-H. Lim, "Ockham's razor in memetic computing: three stage optimal memetic exploration," Information Sciences, vol. 188, pp. 17-43, 2012.

[11] F. Caraffini, F. Neri, G. Iacca, and A. Mol, "Parallel memetic structures," Information Sciences, vol. 227, pp. 60-82, 2013.

[12] H. Ishibuchi, T. Yoshida, and T. Murata, "Balance between genetic search and local search in memetic algorithms for multiobjective permutation flowshop scheduling," IEEE Transactions on Evolutionary Computation, vol. 7, no. 2, pp. 204-223, 2003.

[13] K. C. Tan, S. C. Chiam, A. A. Mamun, and C. K. Goh, "Balancing exploration and exploitation with adaptive variation for evolutionary multi-objective optimization," European Journal of Operational Research, vol. 197, no. 2, pp. 701-713, 2009.

[14] H. A. Abbass, "An evolutionary artificial neural networks approach for breast cancer diagnosis," Artificial Intelligence in Medicine, vol. 25, no. 3, pp. 265-281, 2002.

[15] J. Tang, M. H. Lim, and Y. S. Ong, “Diversity-adaptive parallel memetic algorithm for solving large scale combinatorial optimization problems," Soft Computing, vol. 11, no. 9, pp. 873-888, 2007.

[16] S. M. K. Hasan, R. Sarker, D. Essam, and D. Cornforth, "Memetic algorithms for solving job-shop scheduling problems," Memetic Computing, vol. 1, no. 1, pp. 69-83, 2009.

[17] K. K. Lim, Y.-S. Ong, M. H. Lim, X. Chen, and A. Agarwal, "Hybrid ant colony algorithms for path planning in sparse graphs," Soft Computing, vol. 12, no. 10, pp. 981-994, 2008.
[18] K. C. Tan, C. Y. Cheong, and C. K. Goh, "Solving multiobjective vehicle routing problem with stochastic demand via evolutionary computation," European Journal of Operational Research, vol. 177, no. 2, pp. 813-839, 2006.

[19] A. Caponio, G. L. Cascella, F. Neri, N. Salvatore, and M. Sumner, "A fast adaptive memetic algorithm for online and offline control design of PMSM drives," IEEE Transactions on Systems, Man, and Cybernetics, Part B: Cybernetics, vol. 37, no. 1, pp. 28-41, 2007.

[20] F. Neri and E. Mininno, "Memetic compact differential evolution for cartesian robot control," IEEE Computational Intelligence Magazine, vol. 5, no. 2, pp. 54-65, 2010.

[21] Z. W. Geem, "Parameter estimation for the nonlinear muskingum model using the BFGS technique," Journal of Irrigation and Drainage Engineering, vol. 132, no. 5, pp. 474-478, 2006.

[22] J. Kim, C. S. Kim, and Z. W. Geem, "A memetic approach for improving minimum cost of economic load dispatch problems," Mathematical Problems in Engineering, vol. 2014, Article ID 906028, 11 pages, 2014.

[23] J. Abadie and J. Carpentier, "Generalization of the wolfe reduced gradient method to the case of nonlinear constraints," in Optimization, pp. 37-47, Academic Press, 1969.

[24] O. Yeniay, "A comparative study on optimization methods for the constrained nonlinear programming problems," Mathematical Problems in Engineering, vol. 2005, no. 2, pp. 165-173, 2005.

[25] L. S. Lasdon, A. D. Waren, A. Jain, and M. Ratner, "Design and testing of a generalized reduced gradient code for nonlinear programming," ACM Transactions on Mathematical Software, vol. 4, no. 1, pp. 34-50, 1978.

[26] K. M. El-Naggar, M. R. AlRashidi, M. F. AlHajri, and A. K. Al-Othman, "Simulated Annealing algorithm for photovoltaic parameters identification," Solar Energy, vol. 86, no. 1, pp. 266274, 2012.

[27] J. A. Jervase, H. Bourdoucen, and A. Al-Lawati, "Solar cell parameter extraction using genetic algorithms," Measurement Science and Technology, vol. 12, no. 11, pp. 1922-1925, 2001.

[28] M. R. AlRashidi, M. F. AlHajri, K. M. El-Naggar, and A. K. AlOthman, "A new estimation approach for determining the IV characteristics of solar cells," Solar Energy, vol. 85, no. 7, pp. 1543-1550, 2011.

[29] K. Bouzidi, M. Chegaar, and N. Nehaoua, "New methods to extract the parameters of solar cells from their illuminated IV curve," in Proceedings of the 4th International Conference on Computer Integrated Manufacturing, Sétif, Algeria, 2007.

[30] A. Askarzadeh and A. Rezazadeh, "Parameter identification for solar cell models using harmony search-based algorithms," Solar Energy, vol. 86, no. 11, pp. 3241-3249, 2012.

[31] W. Gong and Z. Cai, "Parameter extraction of solar cell models using repaired adaptive differential evolution," Solar Energy, vol. 94, pp. 209-220, 2013.

[32] M. U. Siddiqui and M. Abido, "Parameter estimation for five- and seven-parameter photovoltaicelectrical models using evolutionary algorithms," Applied Soft Computing Journal, vol. 13, no. 12, pp. 4608-4621, 2013.

[33] Z. W. Geem, "Economic dispatch using parameter-setting-free harmony search," Journal of Applied Mathematics, vol. 2013, Article ID 427936, 5 pages, 2013.

[34] Z. W. Geem, "Parameter estimation of the nonlinear muskingum model using parameter-setting-free harmony search," Journal of Hydrologic Engineering, vol. 16, no. 8, pp. 684-688, 2011. 

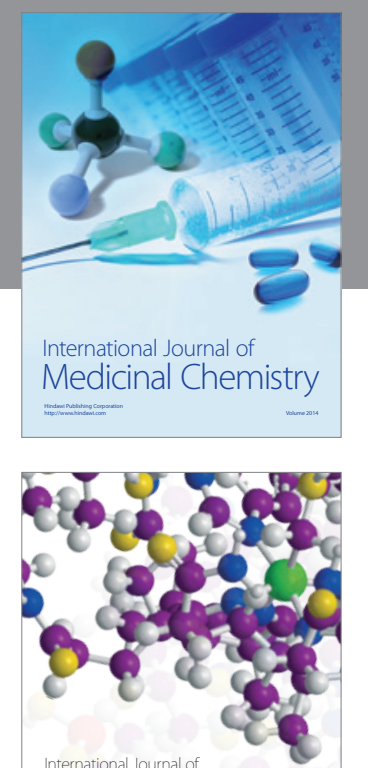

\section{Carbohydrate} Chemistry

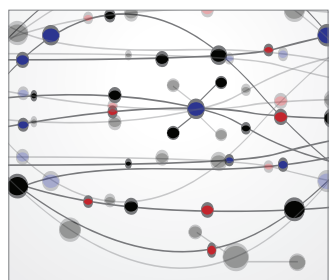

The Scientific World Journal
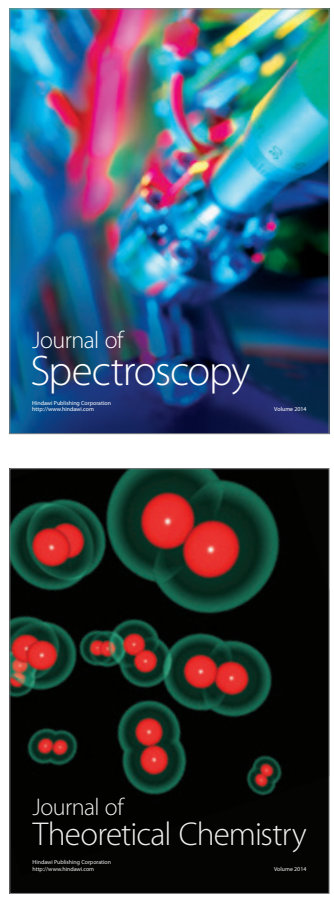
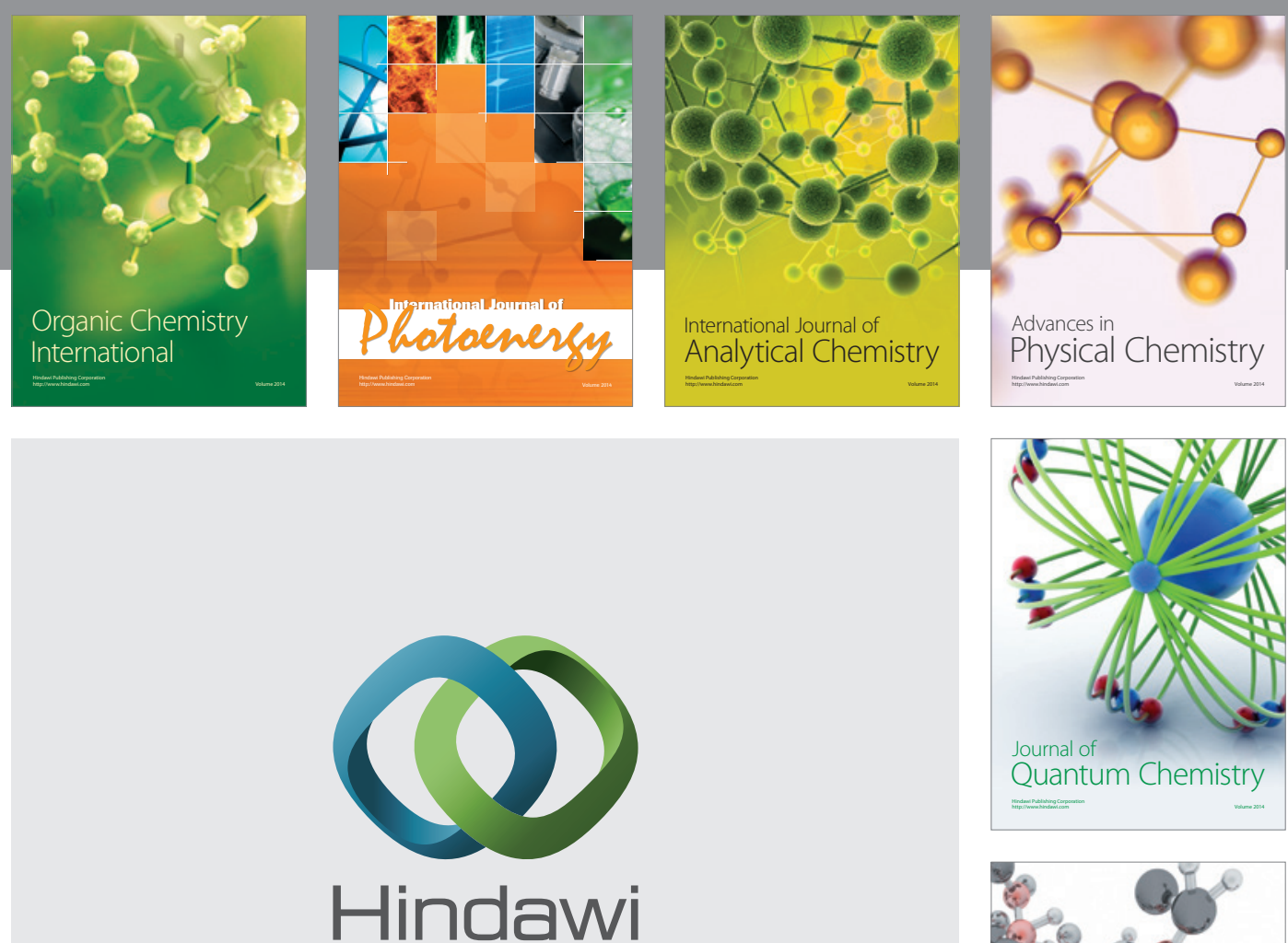

Submit your manuscripts at

http://www.hindawi.com

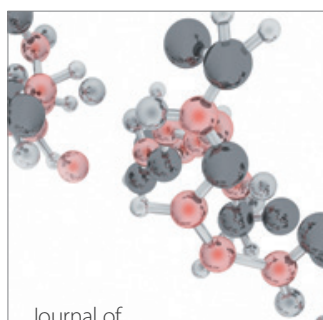

Analytical Methods

in Chemistry

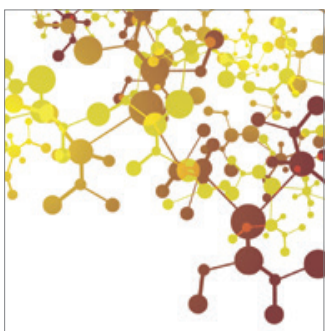

Journal of

Applied Chemistry

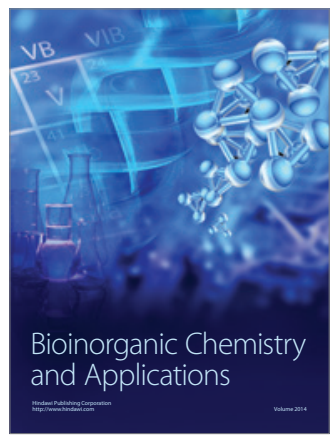

Inorganic Chemistry
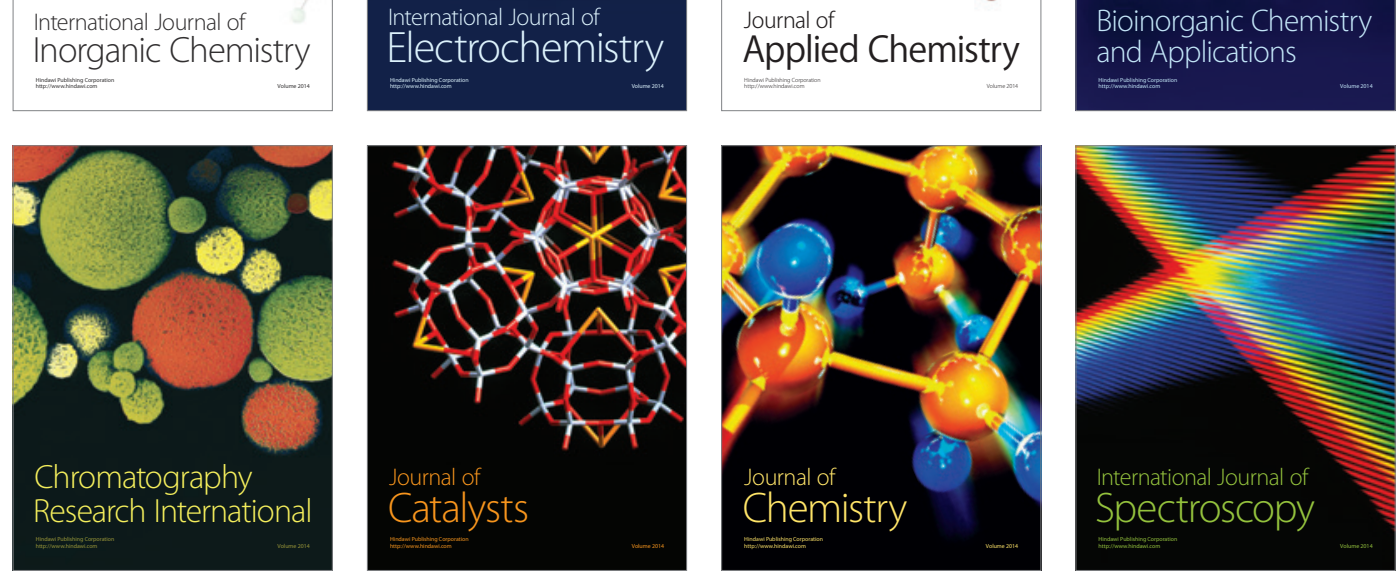